\title{
DESIGNING WIND TURBINE CONDITION MONITORING SYSTEMS SUITABLE FOR HARSH ENVIRONMENTS
}

\author{
D. Ferguson *, V.M. Catterson $^{\dagger}$, C. Booth ${ }^{\Psi}$, A. Cruden $^{T}$ \\ *University of Strathclyde,UK,david.ferguson@strath.ac.uk, ${ }^{\dagger}$ University of Strathclyde,UK,v.m.catterson@strath.ac.uk, \\ ${ }^{\psi}$ University of Strathclyde, UK, campbell.d.booth@strath.ac.uk, ${ }^{T}$ University of Southampton, UK, a.j.cruden@soton.ac.uk
}

Keywords: Wind turbine, condition monitoring system

\begin{abstract}
Research into wind turbine condition monitoring is continually receiving greater attention due to the potential benefits from condition monitoring systems (CMS). These benefits can only be realised with high reliability of the condition monitoring system itself. This paper discusses how CMS reliability can be increased, by introducing four types of robustness and how to design the CMS to meet these requirements. The paper uses a case study CMS installation to illustrate the design requirements, and lessons learned from the installation process.
\end{abstract}

\section{Introduction}

Comprehensive condition monitoring systems (CMS) are beginning to take precedence over the more conventional SCADA systems typically used in the majority of wind turbines. SCADA systems provide 10 minute averaged signals of parameters such as:

- Anemometer-measured wind speed;

- Gearbox lubrication oil temperature;

- Generator winding temperature; and,

- Phase currents.

A SCADA configuration is designed to provide data about operating condition but not necessarily the health of a wind turbine (WT). Where SCADA systems typically provide 10 minute averaged signals, CMS may provide signals in the $\mathrm{kHz}$ range. This higher sampling rate may allow faults or deterioration to be detected at a significantly earlier stage. This can allow maintenance regimes to be optimised and may also allow major failures to be avoided thus reducing the costs associated with wind turbine power production.

For these CMS to be effective in reducing costs and aiding maintenance scheduling, they must provide reliable WT data and be able to operate for long periods of time without any human intervention being required. A CMS which is unreliable and requires significant maintenance resulting in unnecessary visits will only add additional costs to the normal operation of a WT.
This paper will describe the requirements of these CMS in order that they operate reliably for long periods of time in harsh environments; thereby providing economic benefit to the wind farm operator through reduced maintenance and WT downtime. It will discuss the installation of a CMS on a Vestas V47 wind turbine highlighting system requirements and a number of learning outcomes from the installation process. Finally it will discuss some preliminary findings from the data received from the installed system.

\section{CMS Application}

For reliable CMS to be developed detailed analysis of fault origins based on real data has to be carried out. Although simulation or test rig data is readily available it may not necessarily reflect the processes occurring within a WT. In order to obtain this data a comprehensive CMS described in $[1,2]$ has been installed in a Vestas V47 wind turbine on a wind farm in Scotland. It is intended that analysis of this high frequency data will identify patterns of machine degradation. This may allow faults or deterioration to be identified at a significantly earlier stage, allowing maintenance to be carried out more economically and potentially avoiding any major failures.

Since the installed system has been described in detail in [1, 2], only a brief overview of the system will be given here. The CMS itself can be categorised into two main units: the data acquisition unit located in the nacelle and the storage unit located in the base of the tower. The two units are connected via a high speed fibre optic link. The data acquisition unit has two DAQ cards: a high speed card sampling at $20 \mathrm{kHz}$ and a low speed card sampling at $50 \mathrm{~Hz}$. These two cards are connected to a computer which the main LabVIEW program runs on. The program retrieves the data, converts it into a SQL format and sends it to the storage unit via the fibre optic link. The storage unit also has a computer which allows access to the top computer via the remote desktop. The bottom computer has a $2 \mathrm{~TB}$ external network drive which can be switched over with an empty drive once full. Based on the amount of data being stored this drive will be switched over once a month.

The parameters to be measured were selected based on those which are most critical for analysis; these are given below for the high speed and low speed DAQs respectively. 


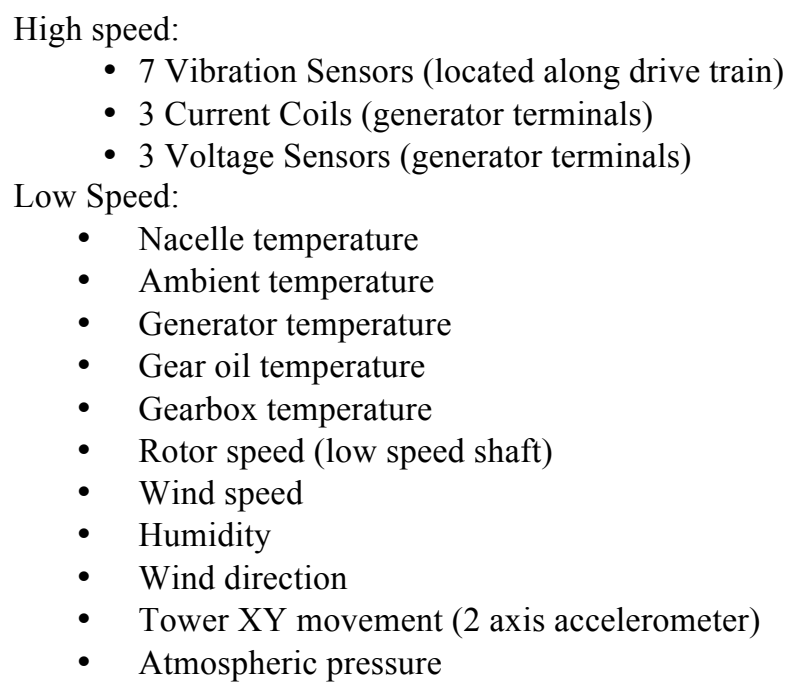

There is also a compass measuring yaw position connected to the data acquisition unit computer via a serial connection.

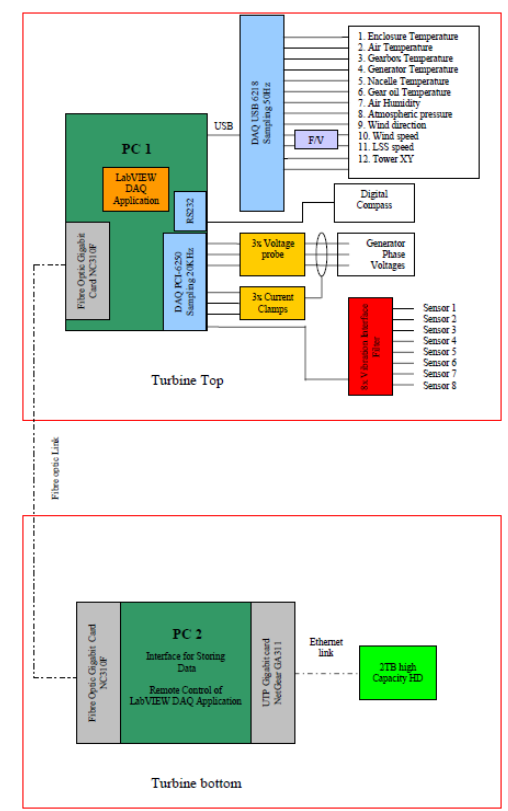

Figure 1: CMS Block Diagram

\section{CMS Robustness}

It has been noted [3] that wind farm operators are wary of blindly adopting CMS without a reasonable economic justification. In an attempt to clarify the benefits of CMS several studies [4-6] have addressed this topic. Yang et al.[7] however, state that further work is required to address CMS' own reliability and value for money. This section will discuss how these systems can be made more reliable by increasing their robustness. It will also discuss how the involvement of third parties can have a significant impact at the installation stage of a CMS deployment.

A system may be considered robust if it is able to function normally in adverse conditions. Wind farms by their nature are located in areas which are inevitably subject to these adverse conditions.

There are four main categories of robustness required:

- Weather robustness;

- Wind turbine operation robustness;

- Handling robustness, and;

- System signal robustness.

It is therefore the role of the system designer or engineer to design a system which is capable of withstanding these conditions.

\subsection{Weather Robustness}

Wind turbines are subject to a wide range of weather conditions all of which must be considered when designing a CMS. Temperatures on a wind farm in Scotland can easily range between -27 and 32 degrees Celsius [8]. These extreme temperatures can have a serious detrimental effect on electrical or moving equipment. The solution to this issue is the use of heaters and fans. Heaters are mainly used on external instrumentation (namely anemometers and wind vanes) and will be built into the device itself. The temperature within the nacelle will be sufficient to protect internal equipment.

Fans are crucial to prevent electrical equipment from overheating. According to [9] generators can reach $150^{\circ} \mathrm{C}$ before a high temperature alarm leads to shut down. This gives a good indication of the temperatures a CMS is exposed to within the nacelle during operation; therefore fans are required to prevent computers and other circuitry from overheating which may result in a system malfunction.

A not uncommon occurrence on wind farms is lightning strikes. A study by the National Renewable Energy Association found that up to 8 out of 100 wind turbines could expect to receive one direct lightning strike every year [10]. To mitigate the damage caused by lightning strikes all wind turbines are fitted with lightning protection which provides a low impedance path to ground. This will shunt the lightning current away from the components susceptible to lightning damage [11]. Equipment fitted on top of the nacelle such as the anemometer or wind vane is very vulnerable to lightning strikes due to their elevated position and therefore must be robust enough to receive a strike. The enclosure of the CMS itself should have a good connection to earth in order to reduce the impact of any current from a lightning strike.

Rainfall and precipitation are other parameters which can have a detrimental impact on electrical equipment within a CMS. If a printed circuit board (PCB) surface was to be contaminated with a conducting material in the presence of moisture and an applied voltage, the result could cause the lowering of resistance between tracks and pads which could lead to corrosion of metals [12]. Similarly any water ingress to internal electrical terminals can cause short-circuits resulting in damage of equipment through over-currents. IEC Standards known as IP ratings classify how well a product or 
system can protect against the intrusion of solid objects, dust and water. Due to field experience, this paper recommends that a CMS located within the nacelle of a turbine has a minimum rating of IP54 which will sufficiently protect it from dust and will protect it against splashing water which may result from a leak in the nacelle.

\subsection{Wind Turbine Operation Robustness}

A CMS must be robust enough to not only withstand harsh weather conditions but also the conditions applied through normal operation of the WT.

A WT during operation will have significant levels of vibration and tower movement. Vibration levels in particular can have a negative impact on components such as connectors as a result of fretting. Fretting occurs when two touching surfaces move relative to one another. Connectors which are fret will have high contact resistance as a result of a build up of debris from base metal that has worn from the connector surface [13]. A high contact resistance on sensor connectors may result in poor quality signals. To avoid fretting, connectors should be used which have contact materials which have a strong ability to resist the formation of insulating films such as oxides so that metallic contact can be maintained. Common metals chosen for this characteristic include gold, copper, tin, and $\mathrm{Sn}-\mathrm{Pb}$ [13].

As discussed previously the temperature within the nacelle of the WT can reach significant levels. This is mainly due to the heat given off by the gearbox and generator during operation. Fans are therefore required within CMS enclosures to ensure operating temperature limits of the individual components are not exceeded. These limits can be determined from manufacturer guidelines and a thermostat can be set accordingly to switch the fan on.

Another operational parameter which must be taken into consideration during the design of CMS is the effect of the WT yaw motion. In the case where cables have to be run from the nacelle to the base of the tower it is crucial that cables can move freely without becoming trapped or being put under tension resulting in a breakage. WT yaw systems typically have a limit of two turns in each direction before they will reset themselves [14]. It is therefore essential that there is enough slack in any cables that are running from the nacelle to the base of the WT so that they are not damaged should the WT yaw to its limit.

\subsection{Handling Robustness}

A CMS, over its lifetime, will be handled many times by anyone working with or around it. This will begin at the testing stage before the system is even installed. Sensor connecters will be connected and disconnected a considerable number of times during the testing process. This makes it essential that the connectors are robust enough and secured tightly enough to cables or harnesses to ensure they do not break or become loose. Following the testing stage the sensors will then be handled during installation further highlighting the need for their robustness. During any maintenance work being carried out on the WT, it is likely that sensors may be knocked or tugged and therefore must be of sufficient build to withstand this.

It is not only connecters but the CMS enclosures also which must be robust. The enclosure will be particularly vulnerable during the installation stage. Initially the system must be transported from where it is built to the WT where it is to be installed, usually by van or $4 \times 4$ truck. Roads or tracks up to wind farms are typically very rough making the CMS enclosure liable to knocks and bumps. Once at the WT the CMS enclosure will have to be winched from the ground up to the nacelle, another stage where the CMS enclosure is vulnerable to being knocked. The main CMS enclosure should therefore be built of a strong resilient material.

\subsection{Signal Robustness}

The final type of robustness required refers to that of the signals within the CMS. For accurate measurements, signals must be relatively free from electrical noise, something which is hard to avoid within a wind turbine nacelle. The two main causes of electrical noise within an electrical signal cable are power cables within the CMS enclosure itself, and the WT generator. The difficulty in keeping signal cables away from power cables and the generator is due to the lack of space within both the CMS enclosure and the WT nacelle. Within the CMS enclosure where possible, signal cables and power cables should be kept separate. Screened cable, which has a layer of earthed wire, should be used to reduce the effect of electrical noise on the signals. The CMS enclosure should, as much as space permits, be kept as far from the generator as possible.

\subsection{Third Party Considerations}

In theory it is straight forward to plan out a project estimating the time required for each stage; however, there are a number of considerations which can easily be overlooked when attempting to install a system in an operational wind turbine.

The first consideration is the sourcing of components. Standard 'off-the-shelf' components usually have set delivery times. However, when ordering a unique component specific to a certain wind turbine or generator, waiting times can dramatically increase. In this case a generator terminal cover, which was required to be modified before allowing the voltage and current sensors to be installed, took four months to be delivered from the manufacturer overseas. Therefore all components whether 'off the shelf' or 'made to order' should be ordered at the earliest opportunity with delays in delivery being incorporated into the project timescale.

Wind farms by their nature are located in remote locations that are subjected to high wind speeds and harsh weather conditions. Firstly in order to access the wind farm roads or tracks must be relatively free from snow and ice; therefore there will be large periods of time during the winter months where access is restricted. Once on the wind farm, in order to climb the wind turbine, for health and safety reasons, the wind speed must be below a certain threshold. The threshold 
set will be specific to the wind farm operator but tends to be around $10 \mathrm{~m} / \mathrm{s}$ based on the authors' experience. Wind farm operators will also avoid, where possible, shutting down the wind turbine during high wind speeds for maintenance or installation work because of the lost revenue that will be incurred. When planning the installation of a CMS it is therefore wise to avoid installing the system during the winter months since lengthy delays can be experienced due to restrictions in accessing the wind turbine.

It was a requirement that installation engineers must be accompanied by two qualified technicians. This requirement imposes another possible delay. Since technicians can only climb WTs to carry out maintenance when the wind speed is below a certain threshold, whenever the wind speed is low enough to climb they have a backlog of work to complete which takes priority over any new installation work. It is therefore wise to include this possible set back into any installation schedule and again beneficial to aim to install during the summer months where wind speeds are generally lower.

\section{Discussion of Preliminary Results}

For the initial month of site operation, CMS data was collected and validated against SCADA data for the same turbine from the same time period. Wind speed and rotor speed measurements are shown in Table 1.

\begin{tabular}{|c|c|c|}
\hline Parameter & SCADA Data & CMS Data \\
\hline Rotor Speed & $28.8 \mathrm{rpm}$ & $28.886 \mathrm{rpm}$ \\
\hline Wind Speed & $9.324 \mathrm{~m} / \mathrm{s}$ & $9.562 \mathrm{~m} / \mathrm{s}$ \\
\hline
\end{tabular}

Table 1: SCADA data compared with CMS data

As stated previously the SCADA data is averaged over 10 minute periods, whereas the CMS data (for these parameters) is measured at 50 samples per second. Therefore, for this comparison, the CMS data for the same 10 minute period was also averaged. It can be seen that the rotor speeds are almost identical, which is to be expected since the WT is operating at constant speed and is not susceptible to large fluctuations. Wind speed on the other hand is more likely to show a greater difference since anemometers are sensitive to spatial variations in the wind therefore resulting in differences between SCADA and CMS readings.

A similar process has been used to validate all other parameters recorded by the CMS. Future work will involve detailed data mining and analysis of the CMS data.

\section{Conclusion}

CMS have the potential to reduce costs of wind turbine maintenance through allowing maintenance to be scheduled more economically and also through the potential avoidance of any major failures. To increase these benefits, CMS must be reliable and not impose any addition maintenance work themselves. They must also provide precise, reliable data which is relatively free from noise. This paper has described how CMS should be built in order to operate reliably for long periods of time in harsh environments.

\section{Acknowledgements}

This research has been funded by the UK Engineering and Physical Sciences Research Council. It is also part of the work being undertaken by the SuperGEN Wind Consortium.

\section{References}

[1] G. Swiszcz, et al., "A data acquisition platform for the development of a wind turbine condition monitoring system," in Condition Monitoring and Diagnosis, 2008. CMD 2008. International Conference on, 2008, pp. 1358-1361.

[2] A. Zaher, et al., Database Management for High Resolution Condition Monitoring of Wind Turbines. New York: IEEE, 2009.

[3] A. Zaher, et al., "Online wind turbine fault detection through automated SCADA data analysis," Wind Energy, vol. 12, pp. 574-593, 2009.

[4] Y. Amirat, et al., "A brief status on condition monitoring and fault diagnosis in wind energy conversion systems," Renewable and Sustainable Energy Reviews, vol. 13, pp. 2629-2636, 2009.

[5] Z. Hameed, et al., "Condition monitoring and fault detection of wind turbines and related algorithms: A review," Renewable and Sustainable Energy Reviews, vol. 13, pp. 1-39, 2009.

[6] D. McMillan and G. W. Ault, "Condition monitoring benefit for onshore wind turbines: sensitivity to operational parameters," IET Renewable Power Generation, vol. 2, pp. 60-72, 2008.

[7] W. Yang, et al., "Wind turbine condition monitoring: technical and commercial challenges," Wind Energy, 2012.

[8] MetOffice. (April2013). Available: http://www.metoffice.gov.uk/climate/uk/extremes/

[9] G. Peng, et al., "Wind turbine generator conditionmonitoring using temperature trend analysis," IEEE Transactions on Sustainable Energy, vol. 3, pp. 124133, 2012.

[10] B. McNiff, "Wind turbine lightning protection project 1999-2001. NREL Subcontractor Report," SR-500-311152002.

[11] B. Glushakow, "Effective Lightning Protection For Wind Turbine Generators," IEEE Transactions on Energy Conversion, vol. 22, pp. 214-222, 2007.

[12] ERA-Technology. (April 2013). Contamination and moisture effects on printed circuit board reliability. Available: http://www.era.co.uk/casestudies/contamination-and-moisture-effects-onprinted-circuit-board-reliability/

[13] M. Antler, "Electrical effects of fretting connector contact materials: A review," Wear, vol. 106, pp. 533, 1985.

[14] LORC. (April 2013). The Yaw System. Available: http://www.lorc.dk/nacelles/yaw-system 